Tilea Brindusa, Brînzaniuc Klara, Tilea I.

\title{
Incidence, epidemiology and etiology of acute meningitis in Mures County - an observational study
}

Clinical County Hospital, Department of Infectious Disease

University of Medicine and Pharmacy of Targu Mures

\begin{abstract}
Background: Meningitis are multiple aetiology disorders characterized by infectious triggered inflammation of the meningeal serrosa manifesting as an acute systemic illness with variable evolution from cure to death. The objectives of this study were the evaluation of incidence, epidemiologic and diagnostic aspects of the acute meningitis in a well define region.

Methods: we have designed an observational retrospective study that included a number of 165 consecutively admitted patients with the diagnosis of meningitis at the Targu-Mures Infectious Disease Clinic, Romania, from January 1st to December 31st 2011. We have compared cases of confirmed meningitis vs. meningismus, and meningeal reaction. We kept track of the demographic aspects, the annual and seasonal distribution of cases. The diagnosis of meningitis was established by examination of the cerebrospinal fluid such as: aspect, pleocytosis, biochemical reactions, wet mounts, Latex agglutination tests, serological reactions, cultures and antibiotic resistance testing (when needed). Results: the majority of the patients were male, from rural areas. The mean age of patients with bacterial
\end{abstract}

\section{Brindusa Tilea}

Clinical County Hospital, Department of Infectious Disease 189 Gh. Doja st., 540225 Targu Mures

Tel.: +40-(0)265-250533

E-mail: brindusa_tilea@yahoo.com meningitis was 36 years old, and that of viral meningitis cases was 20 years old. The acute meningitis incidence in 2011 was 19.73 cases/ 100.000 inhabitants. 26 patients $(15.76 \%)$ of the observed group were diagnosed with bacterial meningitis, and 63 cases $(38.18 \%)$ with viral meningitis. The most common cause for bacterial meningitis were Gram-positive cocci, and for viral meningitis, the varicella-zoster virus.

Conclusions: in 2011, the incidence of acute meningitis in our region has not decreased significantly compared to previous studies. In the case of bacterial meningitis, the etiologic agent was identified in a small proportion, possibly because of antibiotic treatment administered before the admission, also observing an increase in antibiotic resistance of the identified agents.

Keywords: meningitis, epidemiology, incidence, diagnostic.

\section{Background}

Acute infectious meningitis are an important chapter of infectious pathology and also a major public health issue because of the relatively high morbidity and severity by the high mortality rate and by the myriad of neuropsychic sequelae. In the recent years there has been important progress made regarding the pathogenic mechanisms at a molecular level, but establishing the cause of acute viral and bacterial meningitis is still a major concern. Meningitis are the 
most frequent and important infections of the central nervous system, that require emergency diagnosis and treatment. In the preantibiotic era, the prognosis of bacterial meningitis was extremely dire, with more than $90 \%$ mortality. The introduction of antibiotics has significantly influenced their evolution, lowering the mortality for some entities to less than $10 \%[4,5]$.

In the etiology of acute meningitis are involved a number of pathogens (viruses, bacteria, mycobacterium, fungi, protozoa, Chlamydia, Mycoplasma), the latter increasingly affecting the immunodeficient patients in the last decade.

Viral meningitis are the most frequent types in Romania, the most commonly involved organisms being the enteroviruses (Coxsackie, Echo, mumps virus, influenza viruses), their frequency and etiologic profile being influenced by their epidemic evolution [3].

Bacterial meningitis, although secondary from the point of view of their frequency, distinct themselves by their severity, their etiology being variable depending on the physiological and immunological conditions, and the presence of favorable environmental factors. The most frequently isolated pathogens are Meningococcus, Pneumococcus, Gram-negative bacilli, Mycobacterium tuberculosis, Staphylococcus, Leptospira.

Vaccination strategies have substantially changed the epidemiology of community acquired bacterial meningitidis during the past two decades [12].

\section{Methods}

Offering information regarding the incidence of acute infectious meningitis, of meningeal reactions and meningismus, their current etiology (viral, bacterial), and diagnostic and evaluation limits of prognosis factors and the comparison of the obtained results to the current literature. All data were collected retrospectively from patient's medical records. No other procedures were performed specifically for the purposes of this study.

The objectives of the current research were the evaluation of the incidence, epidemiological and diagnosis aspects of acute meningitis.

To this end we have designed an observational retrospective study, which included 165 consecutively admitted patients with the diagnosis of acute meningitis at the Targu-Mures Infectious Disease Clinic, Romania, throughout 2011. In order to facilitate a better analysis we also established a comparison between confirmed meningitis cases and meningismus or meningeal reaction cases.

For the design of the study we have followed:

- the distribution of cases by etiology, sex, age groups, environment, social status

- the cases' frequency throughout 2011

- a comparison between the confirmed meningitis cases, meningismus and meningeal reaction, from the point of view of symptoms and signs, treatment approach and it's influence on the subsequent evolution by quantifying the average hospital stay in days, the results of laboratory tests (inflammatory reactants corroborated with the cerebrospinal fluid results) and the results of cerebral imagery (CT and/or MRI scans).

The diagnosis of acute meningitis was established based on the symptoms and signs (the presence of the following syndromes: infectious, intracranial hypertension and meningeal) confirmed by the alterations in the cerebrospinal fluid CSF (aspect, pleocytosis, biochemical reactions).

The definite diagnosis was established based on the isolation of the etiologic agent from the CSF by means of:

- cultures (Müller-Hinton, blood-gelose, chocolat, or Löwenstein growth media)

- Gram and Ziehl-Neelsen stains

- Latex agglutination tests (for the detection of bacterial antigens)

- blood and CSF serologic tests for bacteria and for the following viruses: enteroviruses, adenoviruses, West Nile virus, herpes simplex virus, mumps virus, influenzae and parainfluenzae viruses, leptospira

- blood cultures using the BactT/Alert system

The statistical analysis and the graphical representations were done using Microsoft $\AA$ Word 
2007, and the statistical computations using GraphPad InStat 3.06 software.

This study was approved by the Ethics Committee of the University of Medicine and Pharmacy at Targu Mures, Romania and has been performed in accordance with standards of the Declaration of Helsinki. Written informed consent was obtained from each patient or legal tutor if the patient was a minor.

\section{Results}

Of the 165 studied cases, admitted under suspicion of acute meningitis, 89 were confirmed by means of paraclinical tests, 60 patients were discharged after the diagnosis of meningismus was established, 15 patients with meningeal reaction, 1 case was diagnosed with radiculitis.

From the biologically diagnosed meningitis, serologically 26 were bacterial and 63 viral (Figure $1)$.

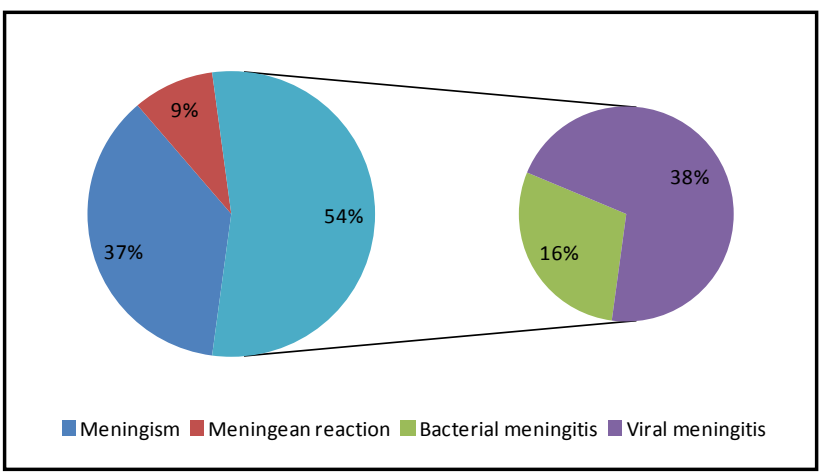

Figure 1 - Case distribution by diagnosis

The incidence of acute meningitis, regardless of their cause, in the study population in 2011 was 19.73 cases/100.000 inhabitants; bacterial meningitis had a cumulated incidence of 6.83 cases $/ 100.000$ inhabitants, and viral meningitis of 12.9 cases $/ 100.000$ inhabitants.
For the studied interval the most affected age group was that of people between 25 and 34 years of age ( 7 bacterial meningitis, 9 viral meningitis, 4 meningismus diagnostics and 2 meningeal reactions), but, almost at the same extent we observed cases in the $1-4$ years old, $5-9$ years old, $10-14$ years old and $35-44$ years old age groups. The age groups with the least number of cases were: under 1 year old, $55-64$ years old and $75-84$ years old (Figure 2).

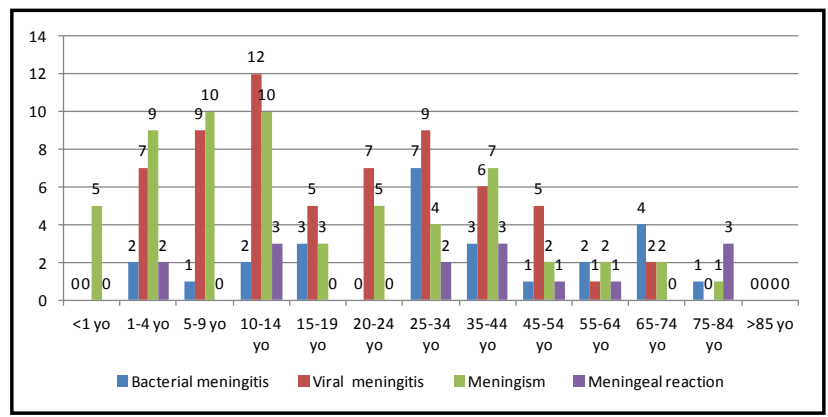

Figure 2 - Case distribution by age and diagnosis

The average age of purulent meningitis was 36 years old, while the average age of viral meningitis cases was 22 years old. The average age of meningismus cases was 20 years old, and that of patients displaying a meningeal reaction was 37 years old.

The incidence of bacterial meningitis was higher for the male population (21, compared to 5 cases for the female population, resulting in a 4.2:1 ratio of male: female meningitis cases); for the viral meningitis cases the ratio was almost equal (1.06 male: female), for meningismus it was 1.4 male: female, and for the patients exhibiting meningeal reactions, the incidence was higher for the female population, the ratio being 0.6 male: female.

The incidence of viral/bacterial meningitis, but also that of meningismus and meningeal reactions was higher in the rural population, the highest difference being observed for the meningeal reactions $(0.15$ urban/rural ratio).

The majority of acute viral meningitis cases where observed in school and preschool aged children (a total number of 37 patients). 
Depending on the month of the admission, the peaks of incidence were registered simultaneously for viral meningitis, meningeal reaction and meningismus in February, April and the August - October period. Most cases were admitted in September. This phenomenon was not observed for bacterial meningitis, in which case the incidence was relatively constant throughout the year (Figure 3).

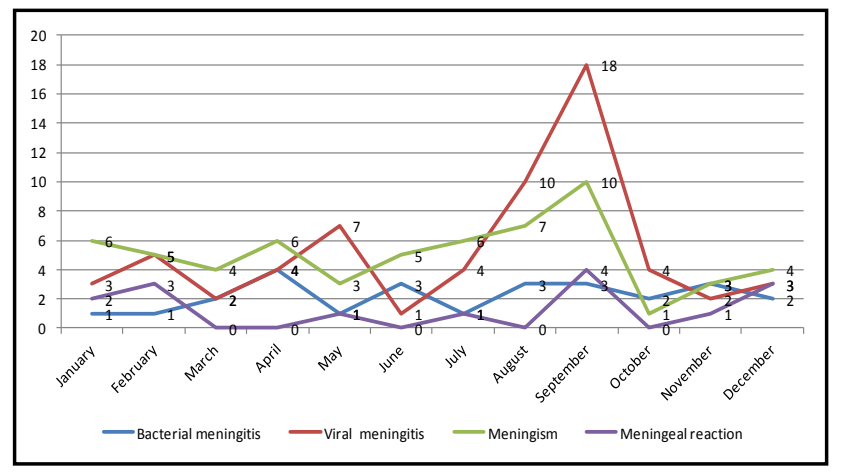

Figure 3 - Relationship between diagnosis and month of admission

The symptoms and signs of the bacterial meningitis cases were dominated by the infectious syndrome ( $100 \%$ of patients), the meningeal irritation syndrome, stiff neck ( $77 \%$ of cases), followed in decreasing order of their frequency by vomiting (65\% of cases); the classical signs of meningeal irritation Kernig I and II, and Brudzinsky I and II were encountered in less than half of these patients.

This frequency distribution was observed in an almost identical manner for the viral meningitis and meningeal reaction cases. But, for the meningismus cases vomiting was prominent (53\%); stiff neck and the classical clinical signs being observed only in approximately $20 \%$ of the cases.

The only clinical sign of meningeal syndrome that is both sensitive and specific at more than $60 \%$ in favor of the meningitis was the neck stiffness $(p<0.0001, \quad$ sensibility $=67 \%, \quad$ specificity $=81 \%$, positive predictive value $=84 \%$, negative predictive value $=62 \%$, with a confidence interval of $95 \%$ ).

Meanwhile, the Kernig and Brudzinsky signs had a higher specificity for the differential diagnosis of meningitis, but a low sensitivity $(\mathrm{p}=0.316$, sensibility $=39 \%$, specificity $=78 \%$, positive predictive value $=72 \%$, negative predictive value $=46 \%$, with a confidence interval of $95 \%$ ).

The presence of vomiting could not be statistically significant associated with either of the conditions $(\mathrm{p}>0.4)$ (Table I).

\begin{tabular}{|c|c|c|c|c|c|c|}
\hline \multirow{2}{*}{ Variable } & \multicolumn{2}{|c|}{ Neck stiffness } & \multicolumn{2}{c|}{ Vomiting } & \multicolumn{2}{c|}{$\begin{array}{c}\text { Kernig/ } \\
\text { Brudzinsky } \\
\text { signs }\end{array}$} \\
\cline { 2 - 7 } & Value & $\begin{array}{c}\mathrm{CI} \\
95 \%\end{array}$ & Value & $\begin{array}{c}\mathrm{CI} \\
95 \%\end{array}$ & Value & $\begin{array}{c}\mathrm{CI} \\
95 \%\end{array}$ \\
\hline Sensitivity & $67 \%$ & $\begin{array}{c}56 \%- \\
77 \%\end{array}$ & $53 \%$ & $\begin{array}{c}40 \%- \\
66 \%\end{array}$ & $39 \%$ & $\begin{array}{c}29 \%- \\
50 \%\end{array}$ \\
\hline Specificity & $81 \%$ & $\begin{array}{c}70 \%- \\
90 \%\end{array}$ & $39 \%$ & $\begin{array}{c}29 \%- \\
50 \%\end{array}$ & $78 \%$ & $\begin{array}{c}65 \%- \\
87 \%\end{array}$ \\
\hline $\begin{array}{c}\text { Positive } \\
\text { predictive } \\
\text { value }\end{array}$ & $84 \%$ & $\begin{array}{c}74 \%- \\
92 \%\end{array}$ & $37 \%$ & $\begin{array}{c}27 \%- \\
48 \%\end{array}$ & $73 \%$ & $\begin{array}{c}58 \%- \\
84 \%\end{array}$ \\
\hline $\begin{array}{c}\text { Negative } \\
\text { predictive } \\
\text { value }\end{array}$ & $62 \%$ & $\begin{array}{c}51 \%- \\
73 \%\end{array}$ & $55 \%$ & $\begin{array}{c}42 \%- \\
68 \%\end{array}$ & $46 \%$ & $\begin{array}{c}36 \%- \\
56 \%\end{array}$ \\
\hline "p" value & $<0.0001$ & \multicolumn{2}{|c|}{0.4} & \multicolumn{3}{c|}{0.0316} \\
\hline
\end{tabular}

The average hospital stay was 12.9 days for bacterial meningitis patients, 11 days for viral meningitis patients, 6.7 days for meningismus cases and 7.2 days for meningeal reaction cases.

As for the co infections or co morbidities associated with the meningeal irritation syndrome, the most frequently associated illness with bacterial meningitis was pneumonia ( $34 \%$ of cases). Acute viral angina was most frequently associated with viral meningitis (44\%) and meningismus (40\%). The majority of acute interstitial pneumonia cases were associated with meningismus (55\%). Intracranial hypertension syndrome (IHT) was most frequent in meningismus cases ( $11 \%$ of meningismus cases).

Regarding the etiology of bacterial meningitis, most cases in which the etiological diagnosis was made, were caused by Streptococcus pneumoniae (6 cases with the average age of 46 years old), followed by methicillin-sensitive Staphylococcus aureus (4 cases with the average age of 33 years old), Neisseria meningitidis (3 cases), Haemophilus influenzae, Klebsiella pneumonia (1 case each), Mycobacterium tuberculosis ( 2 cases) and unsolved etiology (6 cases) (Figure 4). 


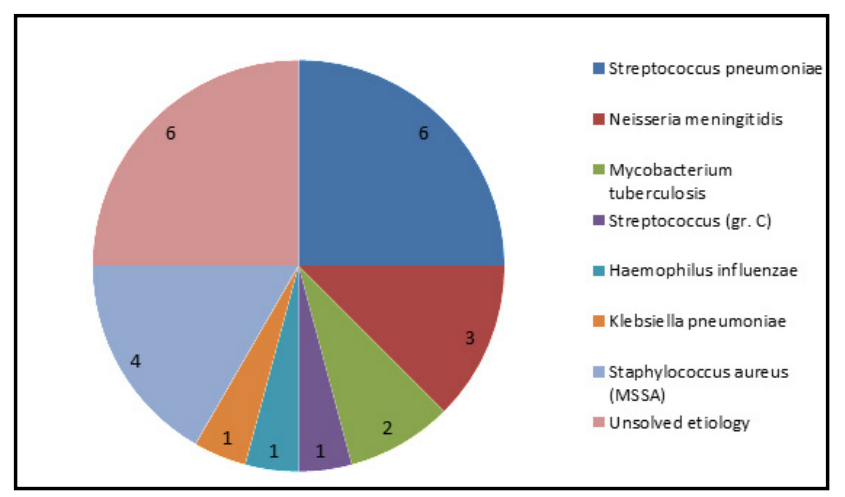

Figure 4 - Distribution of purulent meningitis cases according to the etiologic agent

In 2011, there has been only one confirmed case of meningococcal meningitis in Targu Mures, based on which, if we can try and estimate the incidence of meningococcal meningitis in the city, it would be of 0.75 cases $/ 100.000$ inhabitants.

For the majority of viral meningitis cases we could not identify the causal agent, but for the 12 cases in which the etiologic pathogen was confirmed, they were produced by varicella-zoster virus - VZV ( 7 cases, with an average age of 37 years old), enteroviruses (Echo4 - 4 patients), influenza virus AH1N1 (1 case) (Figure 5).

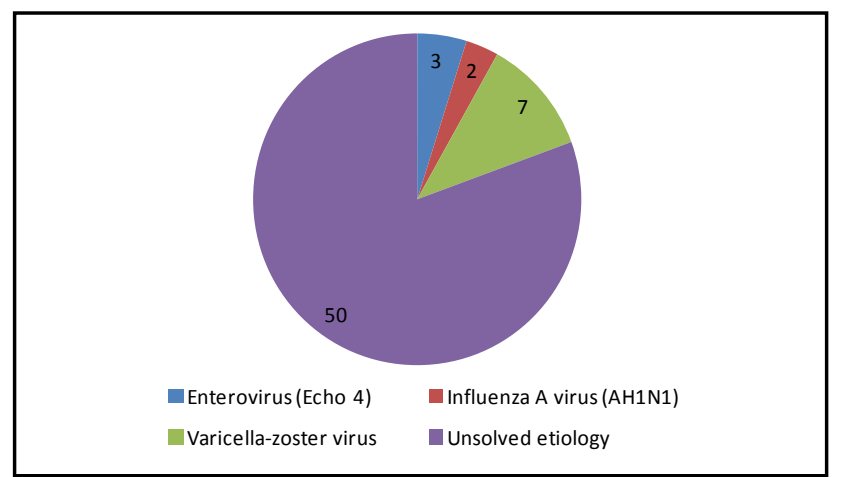

Figure 5 - Distribution of viral meningitis cases according to the etiologic agent

For the quantitative protein content of the cerebrospinal fluid CSF, the highest values (over 200 $\mathrm{mg} / \mathrm{dl}$ ) were seen in patients diagnosed with bacterial meningitis (81\%), and the normal values $(40-45 \mathrm{mg}$ / dl) were seen in meningismus.

Quantitative protein content of the cerebrospinal fluid under $200 \mathrm{mg} / \mathrm{dl}$ was observed in patients with acute viral meningitis (93\% of the cases).

The glucose content of the cerebrospinal fluid exhibited low values $(<45 \mathrm{mg} / \mathrm{dl})$ in a small number of cases; most frequently it was seen in patients with bacterial meningitis ( $26 \%$ of the patients).

After the examination of the macroscopic aspect of the cerebrospinal fluid in patients with bacterial meningitis, 9 patients exhibited clear fluid, 3 patients opalescent fluid, 6 patients xanthochromic and/or hemorrhagic fluid, and 3 patients turbid fluid. For the cases of viral meningitis all the CSF samples were clear. For the patients with meningeal reactions the CSF was clear in the majority of cases ( 5 out of 8 patients).

In the CSF of bacterial meningitis patients the granulocytes were predominant in 12 out of 18 cases, while the lymphocytes were most frequently predominant in viral meningitis cases (24 out of 36 ), and in those with meningeal reaction (7 out of 7 cases) ( Figure 6).

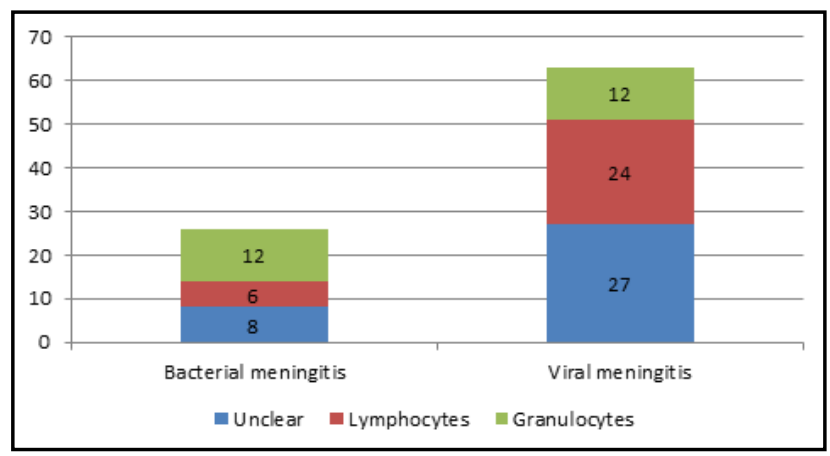

Figure 6 - Distribution of meningitis cases according to the pleocytosis

Imaging techniques did not reveal any alterations specific to meningitis or encephalitis except in half of the cases at the most (4/9 in bacterial meningitis, $5 / 16$ in viral meningitis, $2 / 10$ patients with meningismus and $3 / 6$ patients with meningeal reactions).

Looking at the result of the imaging investigations (cerebral CT/MRI scans), sinusitis, otitis and angina were associated with most cases of 
meningeal syndrome (especially in patients with viral meningitis and meningismus), while cerebral edema was more specific to bacterial meningitis; two cases of Pneumococcus meningitis were associated with acute mastoiditis and sphenoid sinusitis.

\section{Discussions}

The meningitis' incidence in the urban area of Targu Mures, Romania in 2011 was of 19.73 cases/100.000 inhabitants, the viral meningitis having, as expected, an almost double share (12.9 cases/100.000 inhabitants) compared to the bacterial meningitis' incidence (6.83 cases/100.000 inhabitants).

In Romania, on a national scale there is no a statistic database that can specify the cumulative incidence of meningitis (regardless of the etiology), but it is estimated that there are around 15 cases/100.000 inhabitants. This value would place the meningitis' incidence in our area over the national average of the yearly new cases [3].

In one region in Italy (the Lazio region, which includes Rome, with a total population of 5.209.633 in 2000), the meningitis' incidence was, for comparison 3.8 cases $/ 100.000$ inhabitants [10].

In England and Wales, in 2009, the bacterial meningitis' incidence was 3.3 cases $/ 100.000$ inhabitants, showing a decrease from 2005, when there were 4.16 cases/100.000 inhabitants, and from 1999, when there were 7.4 cases/100.000 inhabitants. This reductive tendency was the result of the decreasing incidence of meningococcal meningitis after the introduction of the type $\mathrm{C}$ meningococcal vaccination [15].

In the United States, the incidence of bacterial meningitis in 2007 was 1.8 cases/100.000 inhabitants, down from the previous years. This decrease is also due to anti-meningococcal immunization $[8,9]$.

In sub-Saharan African countries, the very high acute bacterial meningitis' incidence and frequent epidemic outbreaks are mainly due to meningococcus as an etiologic factor, and to several local climate factors that favor the infection, or to better put it increase the general receptivity to the infection [17].

In medical literature it states that yearly variation of bacterial meningitis' incidence is generated especially by the variations of meningococcal infections' incidence, the rest of the etiological factors being irrelevant in the classic epidemiology of infectious disease, the rest of the infections being more likely produced by super infection (pneumococcus, enterobacteria) or iatrogenic interventions (Pseudomonas aeruginosa, Staphylococcus spp, Acinetobacter baumanii) [6].

Still, in our region there has been only one case of meningococcal meningitis in 2011 on a non-vaccinated patient, the other 2 confirmed cases coming from the rural area. So, we cannot discuss a source from the epidemiological sense that would determine this high incidence. Therefore, the cause of the increased incidence that we have observed is due to the rest of etiological agents, that produce a constant number of cases, and that depend on the hygiene and sanitary education level and on the quality of medical care.

Regarding the cases of the present study, the age group with the highest number of meningeal syndrome cases (meningitis, meningismus and meningeal reactions) was the one between 25 and 34 years of age, but, almost at the same extent, we have seen cases in the $1-4$ years old, 5 - 9 years old, $10-$ 14 years old and $35-44$ years old age groups.

In the United States, in 2007, the incidence of bacterial meningitis was higher in the $1-4$ years old group and in the elder population over 50 years old [8].

The same tendency has manifested itself in Great Britain in 2005, where the majority of cases where in the $1-4$ years old age group [15].

In countries where the anti-meningococcal vaccine is already implemented, we can observe a tendency to concentrate the cases in the $1-4$ years old age group, but especially under 2 years old, an age when the vaccine is not effective $[19,20]$.

Before the introduction of the antiHiB vaccine (Haemophilus influenzae type B) in Romania, a large number of bacterial meningitis cases were seen in children under the age of 10 , especially in the 
preschool population. Presently, the number of cases in these age groups has decreased significantly and the incidence has gained a relatively uniform aspect regarding the age groups distribution.

Regarding the cases' distribution by the patients gender, for the bacterial meningitis we can observe a clear dominance of men $(80.7 \%)$, in the viral meningitis a relatively equal number $(51.6 \%)$, while the meningeal reactions are more frequently seen in women $(60 \%)$.

Over $60 \%$ of the meningeal syndrome cases came from the rural area, this fact bringing once again into the spotlight the importance of hygiene and sanitary education in the prophylaxis of meningitis.

Most cases of meningitis were seen in pupils $(31.6 \%)$, thus suggesting the contagious character of this illness in collectivity. The same situation was observed in meningismus where the majority of cases were observed in preschoolers $(33.3 \%)$, but with a high number of cases in pupils' ranks (25.4\%).

The incidence of meningismus was higher in the age group $1-14$ years old, thus suggesting the children's increased predisposition towards this clinical syndrome.

Acute bacterial meningitis had a constant incidence in pupils, employees, unemployed or retired people ( $5-6$ cases in each category), but have been rarely encountered in preschoolers ( 2 cases). This tendency confirms the fact that the infection's mechanism is often by super infection or neglection of an already existing infectious source, more than through man to man transmission.

The peak of viral meningitis in 2011 was in August and September (46.6\% of cases), a tendency that we have also observed in meningismus cases ( $26.9 \%$ of cases). Bacterial meningitis did not display a certain tendency of accumulation in a certain part of the year, the incidence being relatively constant, with an average of 2 cases/month, most of the cases being registered in April (4 cases).

The increased incidence during the warm period of the year is due to enteroviruses meningitis, this type of viruses having a well-known tendency to produce illness more often this time of the year. The explanation actually lies in the transmission path, which is mainly through the fecal-oral route or through contaminated food or water. This reason lies at the foundation of the simultaneous increase in meningismus cases, which appear mainly after viral enterocolitis [1].

The small variability of bacterial meningitis cases depending of the time of the year reduces our possibilities of interpreting this phenomenon, especially because of the lower incidence (3, maximum 4 cases in the months with an increased incidence), that refute an eventual transmission of the infection, these cases not being related spatially (endemic source) or temporally.

The average number of hospital stay days has been 13 days for bacterial meningitis, 11.5 days for viral meningitis and 7 days for the cases of meningismus or meningeal reaction. The fact that meningismus and meningeal reaction benefited from a shorter hospital stay points out the fact that the meningeal irritation syndrome lasted for a short period of time, in comparison to meningitis, being dependent to the severity of it's causing illness, and not to the presence of the pathogen and of the subsequent inflammation of the central nervous system.

In a statistic from 2006, in the United States, regarding the number of hospital admissions and the average hospital stay for meningitis cases in total and split for different etiologies, the average hospital stay for meningitis regardless of their cause was 12.8 days, the average hospital stay for bacterial meningitis was 16.6 days, and for viral meningitis was only 4.1 days. These numbers were compared to the average hospital stay in other infectious disease, which was around 5.4 days. From this one can see the seriousness and lengthy evolution of this type of pathology [16].

Regarding the acute co morbidities, $34.6 \%$ of the bacterial meningitis patients also had pneumonia on the course of their hospital stay, but an increased frequency of pneumonia associated to the meningeal syndrome was also seen in patients with meningismus $(55 \%)$. Most patients with viral meningitis had pharyngitis as an associated illness (44.4\%), while the meningeal syndrome was accompanied by intracranial hypertension syndrome in $11.6 \%$ of the meningismus patients.

Data from United States (2007) revealed that pneumococcal meningitis had the highest incidence of the bacterial meningitis, followed by the one caused by Streptococcus agalactiae and meningococcus 
[8,12]. In Canada in 2001, one can observe an almost identical situation, in which the Pneumococcus meningitis has the highest incidence, followed by streptococcal and staphylococcal meningitis [14]. In countries where anti-Haemophilus influenzae and anti-meningococcal immunization is not introduced, the incidence of Haemophilus and meningococcal meningitis is dominant [11]. Thus, one may say that in our region the causes of bacterial meningitis are almost identical to those seen in countries with a highly developed healthcare and epidemiological surveillance system, even without the mass use of antimeningococcal vaccine.

In the etiology of purulent meningitis there are significant differences between the frequency of causative agents, in relation to age, geographical region and multiannual epidemic variations [18].

In European countries, in relation to age, one may observe that Gram-negative bacilli frequently cause infection for the extreme ages; meningococcus and pneumococcus are predominant in an almost equal percentage in children and adults, while Haemophilus is usually seen only in children 2 months to 15 years of age [3].

The meningitis' incidence caused by certain pathogens is different depending on the geographical region. In North America, meningococcus has a low incidence, while Haemophilus meningitis is more frequent than in Europe and Africa in both children and adults $[1,2]$. In Romania, Haemophilus influenzae is a causative agent only for children's meningitis.

Depending on the epidemic multiannual variations, one may see in the past 40 years, concurrent with a certain decline in purulent meningitis morbidity, a tendency to an increased incidence of Gram-negative and staphylococci, and a persistency of pneumococcal etiology of meningitis [2,3].

For the population studied throughout 2011, most cases of bacterial meningitis, in which the etiological agent was identified were produced by Streptococcus pneumoniae (6 cases), followed by Neisseria meningitidis type B (3 cases) and methicillin-sensitive Staphylococcus aureus (4 cases). Also, there were 2 cases of tuberculosis meningoencephalitis. There were 6 cases in which the causative agent could not be identified, because of either previous treatment, or inability to perform lumbar puncture due to IHT.

In the present study, out of all the cases in which the causal agent has been serologically confirmed (13 out of 63 cases), most were caused by the varicellazoster virus ( 7 cases) and only 3 by enteroviruses.

The complete blood count $\mathrm{CBC}$ did not reveal any specific changes in either of the conditions, bearing a minimal importance in the differential diagnosis of the meningeal syndrome, indicating a single fact: an infectious agent has caused the current illness and the presence of a systemic inflammatory syndrome.

From the biochemical investigations, the C-reactive protein had the lowest sensitivity, with often normal values, even in the case of bacterial meningitis.

The erythrocyte sedimentation rate ESR and the levels of plasmatic fibrinogen had, in turn, constantly high values in the case of bacterial meningitis, but being unspecific in the case of viral meningitis, meningismus, and meningeal reactions.

The qualitative CSF protein content was often positive in bacterial meningitis ( $81 \%$ of cases), but not so sensitive in the case of viral meningitis $(65 \%$ of cases).

The glucose content of the CSF was not such a sensitive determination in the case of bacterial meningitis as one might expect, being under $45 \mathrm{mg} /$ $\mathrm{dl}$ in only $26 \%$ of the samples. Instead, in was under this value in only $3.2 \%$ of the viral meningitis cases. The protein content of the CSF was over $200 \mathrm{mg} / \mathrm{dl}$ in $76 \%$ of the bacterial meningitis cases, and in $93 \%$ of the viral meningitis cases.

Depending on the microscopic aspect of the CSF, as expected, the most varied descriptions appear in bacterial meningitis cases, where one may observe: clear liquid (most of the cases), opalescent in 3 cases, xanthochromic and/or hemorrhagic in 6 cases and turbid in 3 cases. From this one may notice the subjective character of the association between bacterial meningitis and the turbid aspect of the CSF obtained through lumbar puncture. An interesting fact was discovered during the description of the CSF in meningeal reactions, where an opalescent aspect was observed in one of the cases and a xanthochromic aspect in two others. The alterations of the CSF aspect in meningeal reactions is in strict connection 

disorder, which, as it is well known, may also be a cranio-cerebral trauma or an alteration of the homeostasis due to a systemic infection.

As for the cellularity of the CSF, from the cases of bacterial meningitis in which this determination was made, $66 \%$ showed pleocytosis consisting of granulocytes, while for viral meningitis, the lymphocytes were dominant in $66 \%$ of the cases; in meningeal reactions there was a lymphocytic infiltrate in all 7 cases.

The modifications observed following imagery investigations (cranial CT/MRI scans) were relatively rare in bacterial meningitis and even rarer in viral meningitis. The sensitivity of this investigation in diagnosing meningitis has been $36 \%$, its specificity $80 \%$ (resulted by comparison with the meningismus cases), positive predictive value $=81 \%$, negative predictive value $=33 \%$, but there could not be established a statistically significant association between the result of the imagery and the diagnosis of acute meningitis $(p=0.44)$.

As for the sensitivity of bacterial strains involved in the etiology of meningitis we have observed Penicillin G resistance in the case of Streptococcus pneumoniae (3 patients), Neisseria meningitidis group $\mathrm{C}$ (1 patient) and aminopenicillines resistance in the case of Haemophylus influenzae.

This resistance was not at all surprising for us, taking into account the fact that in the other regions of the country and in the neighboring countries the above mentioned germ resistance has been communicated in $40-50 \%$ of the cases. to the physiopathological mechanism of the causative

2. The age group where we have seen the highest incidence of the meningeal syndrome was 25 - 34 years old, a situation specific to countries in which there is an immunization program against the etiologic agents that cause potentially epidemic bacterial meningitis.

3. Most of the meningitis cases, especially bacterial meningitis, were seen in males, in patients coming from a rural setting, and in pupils and students.

4. The peak of viral meningitis' incidence was in the warm summer months, while bacterial meningitis had a constant incidence throughout the year.

5. The etiology of bacterial meningitis was dominated by Gram-positive bacteria.

6. The lower airways infections (acute pneumonia) were associated with bacterial meningitis, and lower airways infections with viral meningitis.

7. The global analysis of the clinical and laboratory data of the present study proves that the diagnosis of neuroinfections is still difficult.

\section{Acknowledgement and Funding}

This paper is partially supported by the Sectorial Operational Program Human Resources Development, financed from the European Social Fund and by the Romanian Government under the contract number POSDRU/89/1.5/S/64109.

\section{References}

1. The meningitis' incidence for the urban population of Targu Mures, and the surrounding area is close to the national incidence, but at the same time, much higher than the one in western countries.
1. Mandell G. L, Bennett J. E \& Dolin R. (2010). Mandell, Douglas, and Bennett's Principles and Practice of Infectious Diseases (pp. 1189-1224). 
Philadelphia PA: Ed. Churchill Livingstone Elsevier.

2. Kasper D. L \& Fauci A. S (2010). Harrison's Infectious Diseases (pp. 303-322). New York: Ed. Mc Graw Hill Medical

3. Voiculescu M. Gh (1990). Boli Infecţioase (pp. 343-371). București: Ed. Medicală

4. Brouwer M.C. \& van de Beek D. (2012). Bacterial Meningitis. Ned Tijdschr Tandheelkd. 119(5), 238-42

5. Adarsh B. (2012). Acute community-acquired bacterial meningitis in adults: An evidence-based review. Cleveland Journal of Medicine. 79(6), 393-400

6. Tang L.M. Chen S.T. \& Hsu W.C. et al. (1999). Acute bacterial meningitis in adults: a hospitalbased epidemiological study. Q J Med. 92, 719725

7. Alireza M. J, Abdolreza E. \& Fresteh A. et al. (2009). Temporal analysis of the incidence of meningitis in the Tehran metropolitan area, 19992005, 2009, Population Health Metrics. 7, 19

8. Thigpen M.C., Whitney C.G. \& Messonier N.E et al. (2011). Bacterial Meningitis in the United States, 1998-2007, $N$ Engl J Med. 364, 20162025

9. Floyd R.F., Federspiel C.F. \& Schaffner W. (1973). Bacterial Meningitis in urban and rural Tennessee. American Journal of Epidemiology. 99(6), 395-407

10. Faustini A, Fano V, \& Sangalli M. et al. (2000). Estimating incidence of bacterial meningitis with capture - recapture method, Lazio Region, Italy. Eur J Epidemiol. 16(9), 843-848

11. Public Health Agency of Canada. International Reports of Meningitis. Retrieved 2007 from http://www.publichealth.gc.ca

12. Van de Beek. (2010). Acute and chronic meningitis. In Cohen J., Opal S.M., Powderly W.G. (Eds.), Infectious Disease, Third Ed (pp. 206-217). Mosby Elsevier

13. Pontones P. et al. (2007). 2005 Indiana report of Infectious Diseases, Ed. Indiana State Department of Health - Epidemiology Resource Center, Indianapolis. Retrieved 2007 from http:// www.in.gov/isdh/23857.htm

14. Agence de la sante publique du Canada
(2005). La meningite bacterienne au Canada: hospitalisations (1994-2001). 31. Retrieved 2005 from www.santepublique.gc.ca

15. HPA Center for Infections. Meningitis and septicaemia disease trends in UK. Retrieved from http://meningitis.org/disease-info/typescauses/trends

16. Holmquist L. (Thomson Reuters), Russo C.A. (Thomson Reuters), Elixhauser A. (AHRQ). (2008). Meningitis-Related Hospitalizations in the United States, 2006. HCUP Statistical Brief \#57. Agency for Healthcare Research and Quality, Rockville, MD. Retrieved 2008 from http://www.hcup-us.ahrq.gov/reports/statbriefs/ sb57.pdf

17. Cuevas E., Perez C. \& Baldasano J.M. et al. Meningitis linked to mineral dust transport in Sahel (Second delivery Report), The International Research Institute for Climate and Society, MACC O-INT WP3.1

18. Muangchana C., Chunsuttiwat S. \& RerksNgarm S. et al. (2009). Bacterial meningitis incidence in Thai children estimated by a rapid assessment tool (RAT), Southeast Asian J Trop Med Public Health. 40(3), 553-562

19. Gonzales E.J., Garcia C.L. \& Alcaide J.J.F. et al. (1997). Eficacia de la vacuna meningococica de polisacaridio capsular del grupo C. Rev Esp Salud Publica. 71(2), 103-126

20. Kupek E., Puricelli R.C.B. \& Westrupp M.H.B. (2001). Effectiveness of a Mass Imunization Campaign Against serogroup C Meningococci in Children in the Federal State of Santa Catarina, Brazil. BJID. 5(6), 324-331 